\title{
The Design and Fabrication of a Single Leakage-Channel Grating Coupler
}

\author{
Ronald L. Roncone, Lifeng Li, Keith A. Bates', and James J. Burke \\ Optical Sciences Center \\ University of Arizona \\ Tucson, AZ 85721
}

Lori Weisenbach, and Brian J. J. Zelinski

Department of Materials Science and Engineering

University of Arizona

Tucson, AZ 85721

\begin{abstract}
We describe the modelling and fabrication of waveguide grating couplers with outcoupling efficiencies into a single diffracted order nearing 100\%. Termed Single LeakageChannel Grating Couplers (or SLCGCs), these devices utilize a high-reflectivity dielectric stack to reflect the out-coupled beam diffracted toward the substrate, back up into the air region where it constructively adds with the beam diffracted into the air region. Computer modelling shows that the branching ratio and the leakage rate can be independently controlled, and that the branching ratio is independent of grating depth and grating period. A SLCGC with a branching ratio of $97.1 \%$ was fabricated using a combination of vacuum evaporation and wet-chemical techniques.
\end{abstract}

Key words: grating coupler, thin film, integrated optics, multilayer waveguide

- Now with Storage Products Line of Business, IBM Corp., Tucson, AZ 85744 


\section{Introduction}

The theoretical tools for understanding and analyzing waveguide grating couplers have been well-established for years ${ }^{1,2}$. In addition, numerous techniques exist for the fabrication of waveguide gratings ${ }^{3.4}$. However, several practical issues have limited the commercialization of devices which incorporate grating couplers. These include an undesirable exponentially decaying output beam profile, wavelength sensitivity, and low input and output coupling efficiencies. The first two issues have received considerable attention in the literature ${ }^{5.9}$. The last issue is the primary topic of this paper.

When used as output couplers, typical symmetric-groove gratings split (via diffraction) the guided mode into two beams, of approximately equal power, one going into the cladding and the other going into the substrate. Generally, during device operation only a single out-coupled beam is utilized. As a result about half of the guided mode energy is wasted. Although prism couplers provide $100 \%$ efficient out-coupling into a single beam, they are impractical for integrated-optic applications due to their bulkiness and non-planar nature.

As a potential solution to this problem, Peng and Tamir ${ }^{10}$ first proposed the use of blazed (or asymmetric groove profile) grating couplers to selectively scatter the modal energy, and showed that these devices can have branching ratios (defined in eqn. 2 in the next section) close to $100 \%$. Many authors ${ }^{11-13}$ have reported on the experimental 
demonstration of blazed gratings in both microwave and optical regimes, with branching ratios of $90-98.4 \%$ having been achieved. Without a doubt, blazing of the grating profile has proven to be an effective technique for fabricating very efficient couplers. However, technological problems persist in generating large groove slopes for near vertical incidence. Moreover (and more importantly), geometric constraints imposed by blazing can create a conflict between a chosen leakage rate, $\alpha$, and the branching ratio. A typical example of this is illustrated with the help of figure 1.

Consider a typical triangular-profile blazed grating as shown in figure 1, which has period $\Lambda$, depth $d_{g}$, and slope angle $\theta$. The leakage rate, (generally given in units of $\mathrm{cm}^{-1}$ ) effectively is the rate at which the energy is coupled out of the guide and thus, it determines the out-coupled beam size. Although $\alpha$ depends on various waveguide parameters, it is most strongly dependent on $d_{g}$, and in fact, saturates beyond a certain $d_{g}$. The branching ratio typically is a single-maximum function of $\theta$, with the maximum branching ratio generally occurring for $\theta=40^{\circ}-60^{\circ}$. The problem arises when the device designer needs to choose an unsaturated value for $\alpha$. In this case, once $\alpha$ is chosen, $d_{\theta}$ becomes fixed. However, the $d_{0}$ needed to give the desired $\alpha$ likely is not the same $d_{g}$ required to give the optimal $\theta$ (and thus the optimal branching ratio). Thus, the interdependence of $\alpha, d_{g}, \theta$, and the branching ratio results in a loss of design flexibility. Moreover, note that (from fig. 1) for grating periods in the $0.20-0.50 \mu \mathrm{m}$ range (typical for integrated optic applications), slope angles of $40^{\circ}-60^{\circ}$ require very deep gratings. 
Waveguide grating couplers with branching ratios as high as $96 \%$ also have been demonstrated using holographically fabricated slanted index modulation volume phase gratings $^{14-16}$ on (or in) a waveguiding structure. However, the long-term stability and mechanical strength of the photosensitive and/or waveguiding materials, in addition to compatibility issues, make the broader application of this technique questionable.

Another recent (and somewhat different) approach ${ }^{17.18}$ which yielded branching ratios of $90 \%$, utilized two gratings of the same period, but different depths, separated by a thin coating, and phase shifted with respect to each other, to create an interference effect of the diffracted orders. While this scheme is quite attractive for certain applications, its implementation in a broad range of material systems and device configurations will likely prove to be difficult.

Recently, Agrawal et al. ${ }^{10}$ proposed placing a dielectric stack on the substrate to reflect light diffracted toward the substrate back up and out of the device, and thus creating highly efficient coupling into a single diffraction order. However, such a device was never demonstrated. The remainder of this paper will examine such a structure in detail, and present design criteria, modelling and fabrication results, and alternate configurations of the basic device. We will show that this flexible design concept allows for easily implemented device configurations with independent control of the branching ratio and leakage rate, and that it also is applicable to variable depth gratings and chirped gratings. In addition, we will describe a device that has been fabricated using a 
combination of vacuum-deposition and wet-chemical techniques. A branching ratio of $97.1 \%$ has been measured for this device.

\section{Grating Coupler Background}

Figure 2 shows a schematic illustration of a typical waveguide grating coupler. The indexes of the cladding, film, and substrate are given respectively by $n_{c}, n_{t}$, and $n_{s}$, while the film thickness is designated as $d_{1}$. The grating period and grating depth are represented by $\Lambda$ and $d_{g}$. In the output coupling configuration shown, a guided wave incident on the grating region is diffracted (or "leaked" out) into two (leakage) channels, one into the substrate and one into the cladding. The diffraction angle, $\theta_{c}$, is related to the waveguide and grating parameters by

$$
n_{c} \sin \theta_{c}=N_{-}+\frac{v \lambda}{\Lambda}
$$

where $v$ is a negative integer (usually -1 ), $\lambda$ is the free-space wavelength, and $N_{\text {eff }}$ is the effective refractive index of a particular guided mode. Generally, grating couplers with symmetric groove profiles are designed to have only two leakage channels, in an effort to eliminate energy loss into undesired diffraction orders. Typically, the branching ratios in these devices range between $40-60 \%$. The branching ratio, $R_{i}$ is defined as the ratio of the power into a particular channel to the sum of the powers into all channels: 


$$
R_{i}=\frac{P_{i}}{\sum_{i} P_{i}} \times 100 \%
$$

For the remainder of this paper, when the term "branching ratio" is used, it is implied that we mean the branching ratio into the cladding (air) region.

To reduce effective losses during output coupling, it is highly desirable to have most or all of the incident energy diffracted into a single leakage-channel. The following section describes a device, which we will refer to as a Single Leakage-Channel Grating Coupler, or SLCGC, to accomplish such a task.

\section{SLCGC Principle of Operation}

The objective of the SLCGC structure shown in Figure 3 is to prevent the loss of energy coupled into the substrate by depositing a high-reflectivity quarterwave dielectric stack between the substrate and film to reflect the out-coupled light back toward the air region where it constructively adds with the light originally coupled into the air region. A simplistic ray-optic representation is shown to demonstrate such operation.

The reflectivity of the dielectric stack, with its alternating high $(H)$ and low $(L)$ refractive index quarterwave layers, ultimately determines the maximum branching ratio which can be achieved in a given device. As the number of stack layers increases, or as the refractive index difference between the $H$ and $L$ layers increases, the reflectivity 
correspondingly increases.

The buffer layer serves a dual purpose. The first is to act as an auxiliary substrate to isolate the film layer from the potentially lossy stack. Since the stack layers generally have high propagation losses (as compared to the film) predominantly due to scattering at the interfaces (which is aggravated by the large index difference between adjacent layers), and/or due to inferior material qualities, an appropriate thickness is necessary to minimize the guided energy propagating within the stack layers, and to avoid perturbation of the desired propagation constant. The second purpose of the buffer layer is to serve as a phase compensation layer to ensure that constructive addition of the two beams occurs in the air region. As will be shown in the next section, the branching ratio can be strongly dependent on the buffer layer thickness.

\section{Design Considerations / Modelling Results}

The theoretical data presented in figures 5-7 are generated by a computer program based on the modal method of Botten et al. ${ }^{20}$ for analyzing plane wave diffraction by a rectangular-groove grating. The conversion, or extension of the diffraction grating theory to a waveguide grating theory is straight-forward". The analysis is rigorous in the sense that the only approximation is an unavoidable matrix truncation during numerical implementation. Further details on the theoretical analysis and numerical modelling of the device (in conjunction with model validation) will appear in a future publication. 
In the device configuration explicitly discussed in this paper, the SLCGC is a multimode structure, capable of supporting many TE and TM modes. Consider a device with a 9-layer stack $(5 \mathrm{H}+4 \mathrm{~L}$ ) of alternating $\mathrm{H}$ and $\mathrm{L}$ quarterwave layers (at $\lambda=0.5145$ $\mu \mathrm{m})$, and having the configuration shown in figure 3, and the parameters shown in table 1. In the numerical modelling, the photoresist (grating) layer is treated as an equivalent uniform medium of thickness $0.100 \mu \mathrm{m}$ and root-mean-square refractive index (between air, $n_{c}=1.00$, and photoresist, $n_{g}=1.66$ ) of 1.370 .

Table 2 shows the effective indexes at $\lambda=0.5145 \mu \mathrm{m}$ for all the TE modes of the structure. The electric field intensity profiles for the $T E_{3}$ and $T E_{4}$ modes are shown in Figure 4. The high-loss $T E_{0}-T E_{2}$ modes have nearly all of their energy propagating within the stack layers, and thus are not of significant interest here. Note that the $T E_{3}$ and $T E_{4}$ modes have rather similar mode profiles. Based on the modelling of numerous different device configurations with varying parameters, the similarity in these mode profiles appears to be much more a special case than the general case (but happens to be the case for the device we have fabricated). The $T E_{4}$ mode is the desired mode in this structure because more of its energy is confined in the low-loss film layer.

In addition to mode profile considerations, the designer must be careful to ensure that the effective indexes (and thus the coupling angles) of adjacent modes ( $T E_{3}$ and $T E_{4}$ in this case) are adequately separated to prevent inadvertent launching of the wrong mode. The easiest parameter to adjust for this purpose is film thickness. 
The choice of the number of $\mathrm{H}-\mathrm{L}$ layers to include in the stack is largely dependent upon the desired branching ratio, and the indexes of the materials to be used. As the number of layers in the stack increases, the stack reflectivity (and thus the branching ratio) correspondingly increases. The stack reflectivity is both polarization and angle dependent, and thus the grating period (which effectively controls the angle of the light incident on the stack) and the polarization state of the propagating mode become important considerations. The center wavelength of the reflectance/transmittance spectrum of the stack is chosen to coincide with the operating wavelength of the device.

The branching ratio as a function of buffer layer thickness is shown in Figure 5, for devices having either 3-layer or 9-layer stacks. Note the following:

1. The maximum achievable branching ratio depends on the number of layers in the stack, $(99.2 \%$ for the 9 -layer, and $87.4 \%$ for the 3 -layer) and is periodic with buffer layer thickness.

2. The branching ratia becomes nearty independent of the buffer layer thickness over a broad range, as the number of stack layers increases. This considerably eases the fabrication tolerances on index and thickness control of the individual layers.

3. Although not explicitly shown in the plot, the minimum (practical) buffer layer thickness is dictated by material quality considerations. As $d_{B}$ decreases, more 
guided energy (from the desired mode) is propagated in the stack, and guidedmode losses increase. For example, with the materials we used, a minimum buffer layer of $-0.42 \mu \mathrm{m}$ was necessary before losses stabilized to $2 \mathrm{~dB} / \mathrm{cm}$ for the desired $\mathrm{TE}_{4}$ mode.

Figure 6 and Figure 7 illustrate the flexibility and potential utility of the general design concept. Figure 6 shows the behavior of the branching ratio and the leakage rate, as a function of grating depth, while figure 7 shows the branching ratio as a function of grating period. A buffer thickness of $0.42 \mu \mathrm{m}$ is assumed for both plots. Notice that in both cases, the branching ratio effectively is independent of the grating parameters. This has two important implications:

1. The design concept allows for independent control of both the leakage rate (which is strongly dependent on grating depth) and thus of out-coupled beam size, and the branching ratio.

2. Because the branching ratio is insensitive to the grating parameters, the design concept also should work with variable groove depth and chirped (variable groove spacing) gratings. As a result, high branching ratios can be engineered into grating devices which also possess beam-shaping and focussing capabilities. 


\section{SLCGC Fabrication / Results}

A successful device exhibiting a $97.1 \%$ branching ratio into air has been fabricated using a combination of vacuum-evaporation and wet-chemical (sol-gel) techniques. A 9layer stack, with alternating quarterwave layers of $\mathrm{TiO}_{2}\left(n_{\mathrm{H}}=2.38\right)$ and $\mathrm{SiO}_{2}\left(n_{\mathrm{L}}=1.46\right)$, and a center wavelength of $\lambda=0.5145 \mu \mathrm{m}$, was deposited on a $2^{n} \times 1{ }^{n} \times 1 \mathrm{~mm}$ fused silica substrate using vacuum evaporation ${ }^{22}$. After sonic cleaning the stack for $5 \mathrm{~min}$ in pure, dehydrated ethanol to prepare the surface for further processing, the stack was fired at $500^{\circ} \mathrm{C}$ for $30 \mathrm{~min}$. The turnace bake was performed in an attempt to further densify the structure, and to minimize microstructural changes and stresses in the stack incurred during the subsequent heat-processing of the sol-gel buffer and film layers. The $500^{\circ} \mathrm{C}$ heat treatment shifted the stack spectrum $-10 \mathrm{~nm}$ toward the blue, probably due to a slight densification of the materials. Figure 8 shows the measured reflectance spectrum of the post-bake 9-layer stack, at a $5^{\circ}$ angle of incidence.

The buffer layer and film layer were fabricated using wet-chemically derived solutions ${ }^{23.24}$ spin-coated in a class 100 clean-room environment. The buffer layer was a 13:87 mol\% $\mathrm{TIO}_{2}: \mathrm{SiO}_{2}$ solution, yielding amorphous glass films of refractive index 1.49 . To achieve the desired buffer layer thickness, two layers were deposited. The film layer was fabricated by spin-coating a $35: 65$ mol\% $\mathrm{TiO}_{2}: \mathrm{SiO}_{2}$ solution which yielded an amorphous glass film of refractive index 1.663 and thickness $0.20 \mu \mathrm{m}$ (in a single layer). Each sol-gel layer was baked at $500^{\circ} \mathrm{C}$ for $30 \mathrm{~min}$. Using these materials and buffer 
layer thicknesses above $-0.42 \mu \mathrm{m}$, the loss of the $T E_{4}$ mode was $2.0 \mathrm{~dB} / \mathrm{cm}$ at $\lambda=0.5145$ $\mu \mathrm{m}$. As a comparison, the loss of the $T E_{3}$ mode was $6.8 \mathrm{~dB} / \mathrm{cm}$ (recall the field profiles of figure 4). Entire 5 and 7-layer SLCGCs have been fabricated using the sol-gel materials, but these results are reported elsewhere ${ }^{24}$. It is interesting to note that the entire structure can be fabricated in only two materials, and by using any of the common thin-film deposition methods, or combinations thereof (as long as the materials are of sufficiently low loss).

The $0.30 \mu \mathrm{m}$ period grating, designed to give a $-10^{\circ}$ angle for the out-coupled beams, was fabricated using a holographic technique. Shipley 1805 positive photoresist was diluted 1:1 with Shipley type $P$ thinner, to yield $0.1000 \mu \mathrm{m}$ deep coatings (on top of the multilayer structure) when spun at $5800 \mathrm{~mm}$ for $30 \mathrm{sec}$. The grating exposure was carried out using the $0.4579 \mu \mathrm{m}$ line of an etalon-tuned Argon-ion laser. After this first exposure, a transparent mask (on a $2^{\prime \prime} \times 1^{\prime \prime} \times 1 \mathrm{~mm}$ glass slide) with two rectangular (15 mm $\times 4 \mathrm{~mm}$ ) opaque aluminized regions separated by $1 \mathrm{~cm}$, was placed against the exposed photoresist grating. The resulting mask/grating sandwich was held in front of a xenon-arc lamp for 60 sec to completely expose all regions of the photoresist, except for the two rectangular regions, and thus to delineate an input and output grating. At this point, an in-situ developing technique ${ }^{25}$ was utilized to ensure that the resulting gratings were developed down to the film. The entire structure then was baked at $110^{\circ} \mathrm{C}$ for $30 \mathrm{~min}$ to harden the photoresist gratings. 
To test the SLCGC, the $0.5145 \mu \mathrm{m}$ line of an Argon-ion laser was run through a Glan-Thompson polarizer to give a TE polarized beam, and then gently focused (using a $200 \mathrm{~mm}$ focal length plano-convex lens) onto the input grating. The SLCGC was rotated and translated until the $\mathrm{TE}_{4}$ mode was optimally coupled in. A Si PIN photodiode, connected to a picoammeter, was placed $5 \mathrm{~mm}$ from the output grating (first on the air side, and then on the substrate side) to capture the out-coupled light and convert it to a current. Care was taken to reduce background radiation. The resulting current on the picoammeter was used to calculate the branching ratio as follows:

$$
R_{a t r}=\frac{I_{d b}}{I_{a b}+I_{m b}} \cdot 100 \%
$$

where $\mathrm{I}_{\mathrm{ain}}$ and $\mathrm{I}_{\text {sut }}$ are the currents generated in the photodiode by the out-coupled light. Using this procedure, an average (over several locations on the device) branching ratio of $97.1 \%( \pm 1 \%)$ was measured. The theoretical branching ratio from Fig. 5 (indicated by the ${ }^{\prime \prime} x^{n}$ ) is $96.2 \%$.

Considering the somewhat limited accuracy with which we know the buffer layer thickness $( \pm 0.02 \mu \mathrm{m})$, and that the theoretical design point shown by the " $x^{n}$ in figure 5 is not on a flat region of that plot, the agreement between the theoretical and experimentally measured branching ratio is surprisingly good. While this single sample is insufficient to validate the computer model, it does demonstrate the validity and usefulness of the design concept. Work continues in the fabrication of more devices in 
order to obtain enough data points to validate the model.

\section{Future Applications}

By extending the work reported in this paper, several interesting and potentially useful device configurations can be envisioned:

- As mentioned previously, the basic SLCGC design can be combined with variable depth gratings to form efficient couplers with beam-shaping capabilities, or with chirped gratings to form efficient focusing elements.

- Although only a simple broadband quarterwave stack design was utilized here, the reflecting stack easily could be designed to pertorm filtering and/or polanizing functions as well.

- Instead of placing the buffer layer and reflector under the guiding film and grating, they could be placed on top of the grating region. This would allow for the preferred single-mode device operation, while retaining a high branching ratio in structures where it is not possible to place the stack between the substrate and guiding region, such as in ion-exchanged or ion-diffused waveguides. 


\section{Conclusions}

We have modelled the characteristics of the SLCGC proposed by Agrawal et al. ${ }^{19}$ and have fabricated the device using vacuum deposition and wet-chemical techniques. The branching ratio of the SLCGC has been measured to be $97.1 \%$, much greater than the typical values of $40-60 \%$ exhibited by unmodified output couplers. Modelling indicates that the SLCGC branching ratio is independent of grating depth and period, but depends upon the buffer layer thickness when the number of layers in the reflective stack is small. As the number of stack layers increases, the branching ratio becomes independent of the buffer layer thickness over a broad range. These modelling results indicate that fabrication tolerances on the layer and grating components of the SLCGC are quite broad. They also indicate that the SLCGC design concept can be applied to more sophisticated grating applications including focussing and beam-shaping elements.

\section{Acknowledgements}

We express thanks to John O'Kelly, and Detrick Branston for their work with the sol-gel solution preparation and characterization. We also thank the Donnelly Corporation for performing the spectral characterization of the dielectric stacks. This work is sponsored in part by the Optical Data Storage Center at the University of Arizona. R.L. Roncone acknowledges the financial support of a United States Department of Education GAANN fellowship. 


\section{REFERENCES}

1) T. Tamir (ed.): Integrated Optics, Topics in Applied Physics 7, (Springer, New York, Heidelberg, Berlin 1975), Chap. 3.

2) T. Tamir and S.T. Peng, "Analysis and Design of Grating Couplers," Appl. Phys. 14, 235-254 (1977).

3) M.L. Dakss, L. Kuhn, P.F. Heidrich, and B.A. Scott, "Grating coupler for efficient excitation of optical guided waves in thin films," Appl. Phys. Lett. 16, 523-525 (1970).

4) M. Xu, R. Moshrełzadeh, U.J. Gibson, G.I. Stegeman, and C.T. Seaton, "Simple method for fabricating guided-wave gratings," Appl. Opt. 24, 3155-3161 (1985).

5) S. Miyanaga, and T. Asakura, "Intensity Profile of Outgoing Beams From Uniform and Linearly Tapered Grating Couplers," Appl. Opt. 20, 688-695 (1981).

6) A. Saad, H. Bertoni, and T. Tamir, "Beam Scattering by Nonuniform Leaky-Wave Structures," Proc. of IEEE 62, 1552-1561 (1974).

7) L. Li, K.A. Bates, J.K. Erwin, R.L. Roncone, and J.J. Burke, "Planar Waveguide Grating Couplers With Variable Depth," in OSA Annual Meeting Technical Digest, 
1991 (Optical Society of America, Washington, D.C., 1991), vol 17, p. 26.

8) K.E. Spaulding and G.M. Morris, "Achromatic Waveguide Input/Output Coupler Design," Appl. Opt. 30, 1096-1112 (1991).

9) M.C. Gupta and L. Li, "Achromatic Compensation for Integrated Optic Grating Couplers With Focused Beams," Appl. Opt. 30, 1461-1463 (1991).

10) S.T. Peng and T. Tamir, "Directional Blazing of Waves Guided by Asymmetrical Dielectric Gratings," Opt. Commun. 11, 405-409 (1974).

11) T. Aoyagi, Y. Aoyagi, and S. Namba, "High-Efficiency Blazed Grating Couplers," Appl. Phys. Lett. 29, 303-304 (1976).

12) A. Gruss, K.T. Tam, and T. Tamir, "Blazed Dielectric Gratings with High BeamCoupling Efficiencies," Appl. Phys. Lett. 36, 523-525 (1980).

13) Q. Gong, Input and Output Waveguide Grating Couplers: Theory, Fabrication, and Testing, Ph. D. dissertation, University of Arizona (1990).

14) H. Kogelnik, and T.P. Sosnowski, "Holographic Thin Film Couplers," Bell Syst. Technol. J. 49, 1602-1608 (1970). 
15) W. Driemeier, "Coupled-Wave Analysis of the Bragg Effect Waveguide Coupler," J. of Mod. Opt. 38, 363-377 (1991).

16) W. Driemeier, "Bragg-Effect Grating Couplers Integrated in Multicomponent Polymeric Waveguides," Opt. Lett. 15, 725-727 (1990).

17) I.A. Avrutsky, A.S. Svakhin, and V.A. Sychugov, "Interference Phenomena in Waveguides with Two Corrugated Boundaries," J. of Mod. Opt. 36, 1303-1320 (1989).

18) I.A. Avrutsly, A.S. Svakhin, V.A. Sychugov, and O. Parriaux, "High-Efficiency SingleOrder Waveguide Grating Coupler," Opt. Lett. 15, 1446-1448 (1990).

19) N. Agrawal, T.C. Strand, and P. May, "High Directivity Waveguide Grating Couplers for Optical Storage," CLEO Proc., 1990, 40.

20) L.C. Botten, M.S. Craig, R.C. McPhedran, J.L. Adams, and J.R. Andrewartha, "The Dielectric Lamellar Diffraction Grating," Optica Acta 28, 413-428 (1981).

21) M. Neviere, "The Homogeneous Problem," in Electromagnetic Theory of Gratings, ed. R. Petit (Springer-Verlag, Berlin, 1980). 
22) Stacks provided by: Evaporated Coatings, Inc., 2365 Maryland Road, Willow Grove, PA 19090.

23) L. Weisenbach, B.J.J. Zelinski, J. O'Kelly, J. Morreale, R.L. Roncone, and J.J. Burke, "The Influence of Processing Variables on the Optical Properties of $\mathrm{SiO}_{2}-\mathrm{TiO}_{2}$ Planar Waveguides," to be published in SPIE Proc. of OE/Fibers 1991.

24) L. Weisenbach, B.J.J. Zelinski, R.L. Roncone, L. Li, and J.J. Burke, "Wet-Chemical fabrication of Single Leakage-Channel Waveguide Grating Couplers," submitted to Thin Solid Films, Feb., 1992.

25) L. Li, M. Xu, G.I. Stegeman and C.T. Seaton, "Fabrication of photoresist mask for submicrometer surface relief gratings," Proc. SPIE 835, 72-82 (1987). 


\section{FIGURE CAPTIONS}

Figure 1 - Configuration of a typical triangular-groove profile blazed grating.

Figure 2 - Schematic illustration of a symmetric-groove profile waveguide grating coupler, and it's basic operation.

Figure 3 - Ray-optic schematic illustration of a 9-layer stack SLCGC, and it's principle of operation for achieving high branching ratios.

Figure 4 - Transverse electric field intensity profiles for the device illustrated in figure 3 , and with the parameters given in Table 1, for the $T E_{3}$ (dashed line) and $T E_{4}$ (solid line) guided modes. The vertical lines represent the boundaries between adjacent layers. The waveguiding layer is centered at 0.0 . The horizontal axis is in units of $\mu \mathrm{m}$.

Figure 5 - Plot of branching ratio (\%) vs. buffer layer thickness $(\mu \mathrm{m})$ for devices having 3 layers (dashed line) and 9-layers (solid line) in the dielectric stack. Devices having 5 and 7 layer stacks fall between these two plots. The ' $x$ ' indicates the point where the 9-layer stack device falls. 
Figure 6 - Plot of branching ratio $(\%)$ and leakage rate $\left(\mathrm{cm}^{-1}\right)$ vs. grating depth $(\mu \mathrm{m})$, for a 9-layer stack SLCGC. The solid line is the branching ratio. The dashed line is the leakage rate. The arrows indicate which vertical axis corresponds to each curve. Note that branching ratio effectively is independent of both grating depth, and leakage rate.

Figure 7 - Plot of branching ratio (\%) vs. grating period $(\mu \mathrm{m})$, for a 9-layer stack SLCGC, showing that branching ratio effectively is independent of the grating period.

Figure 8 - Plot of measured reflectance vs. $\lambda$ for a 9-layer stack, after baking at $500^{\circ} \mathrm{C}$ for $30 \mathrm{~min}$. Peak reflectivity is $97.5 \%$ in the range $500-507 \mathrm{~nm}$.

Table 1 - Modelling and fabrication parameters for the SLCGC structure shown in figure 3.

Table 2 - Computed effective indexes for the TE modes (at $\lambda=0.5145 \mu \mathrm{m}$ ) of a nine-layer stack SLCGC (shown in figure 3) having the parameters listed in table 1. 


$$
\begin{array}{ll}
n_{3}=1.461 & \\
n_{H}=2.380 & d_{H}=0.0525 \mu \mathrm{m} \\
n_{L}=1.460 & d_{L}=0.0856 \mu \mathrm{m} \\
n_{B}=1.49 & \\
n_{1}=1.663 & d_{1}=0.200 \mu \mathrm{m}
\end{array}
$$$$
\Lambda=0.30 \mu \mathrm{m} \quad \lambda=0.5145 \mu \mathrm{m}
$$ 


Mode $\quad N_{\text {off }}$

\begin{tabular}{ll}
$\mathrm{TE}_{0}$ & 1.8870 \\
$\mathrm{TE}_{1}$ & 1.8204 \\
$\mathrm{TE}_{2}$ & 1.7056 \\
TE $_{3}$ & 1.5460 \\
TE $_{4}$ & 1.5328 \\
\hline
\end{tabular}

Table 2 


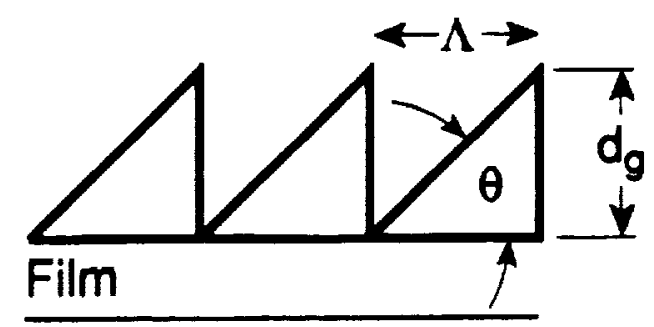

Substrate

Fig. 1

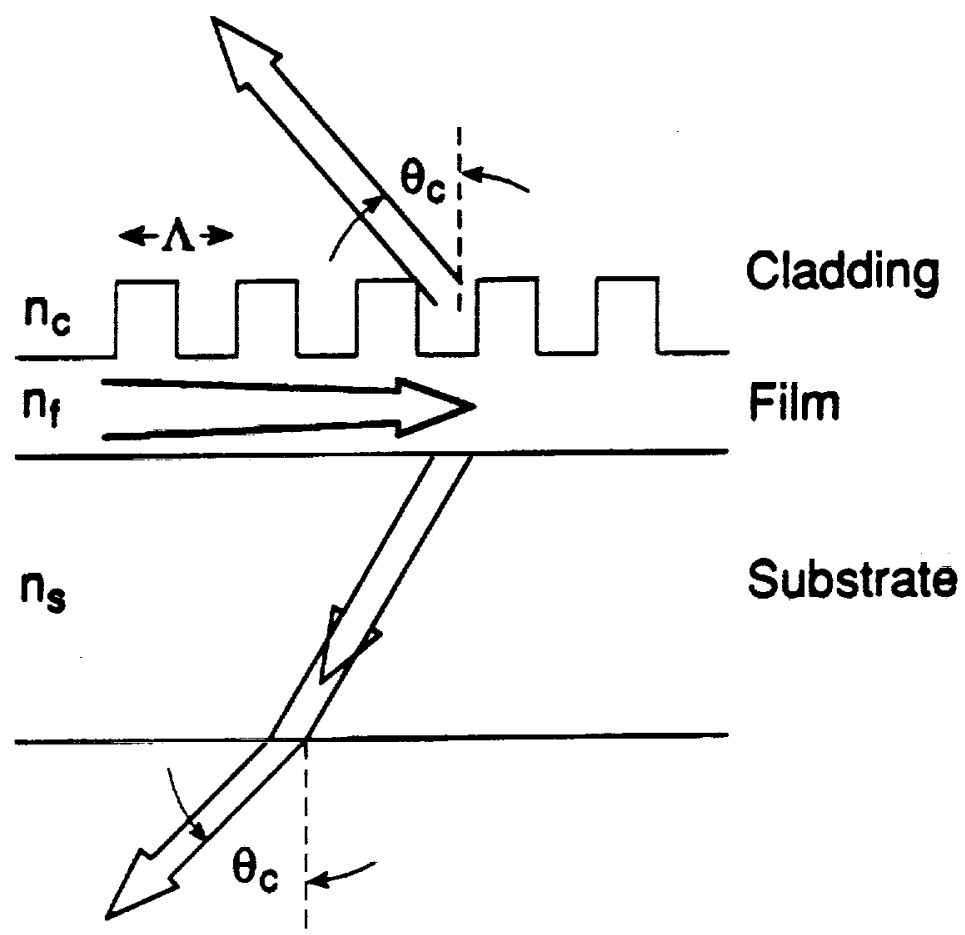

Fig. 2 


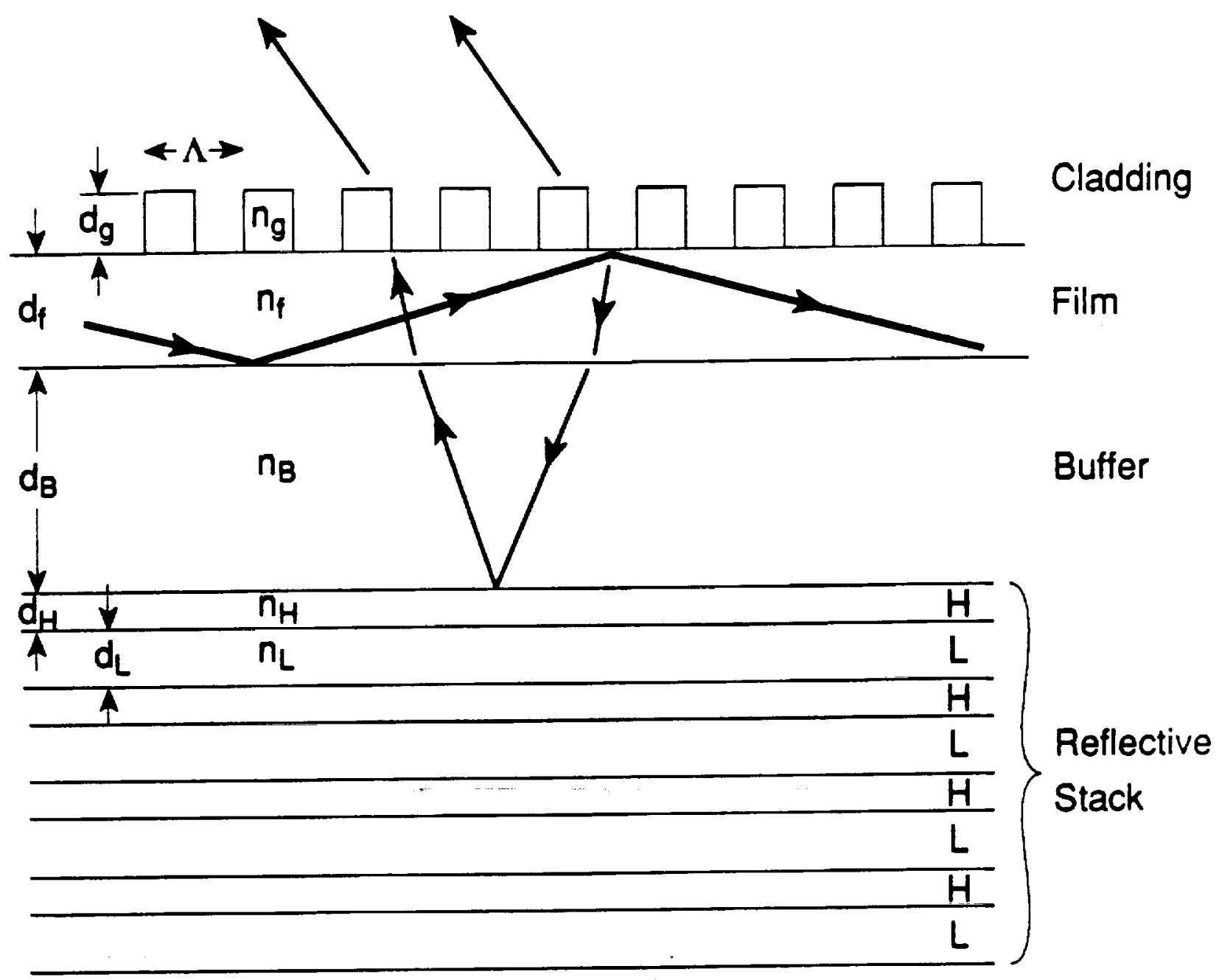

Substrate

Fig. 3 


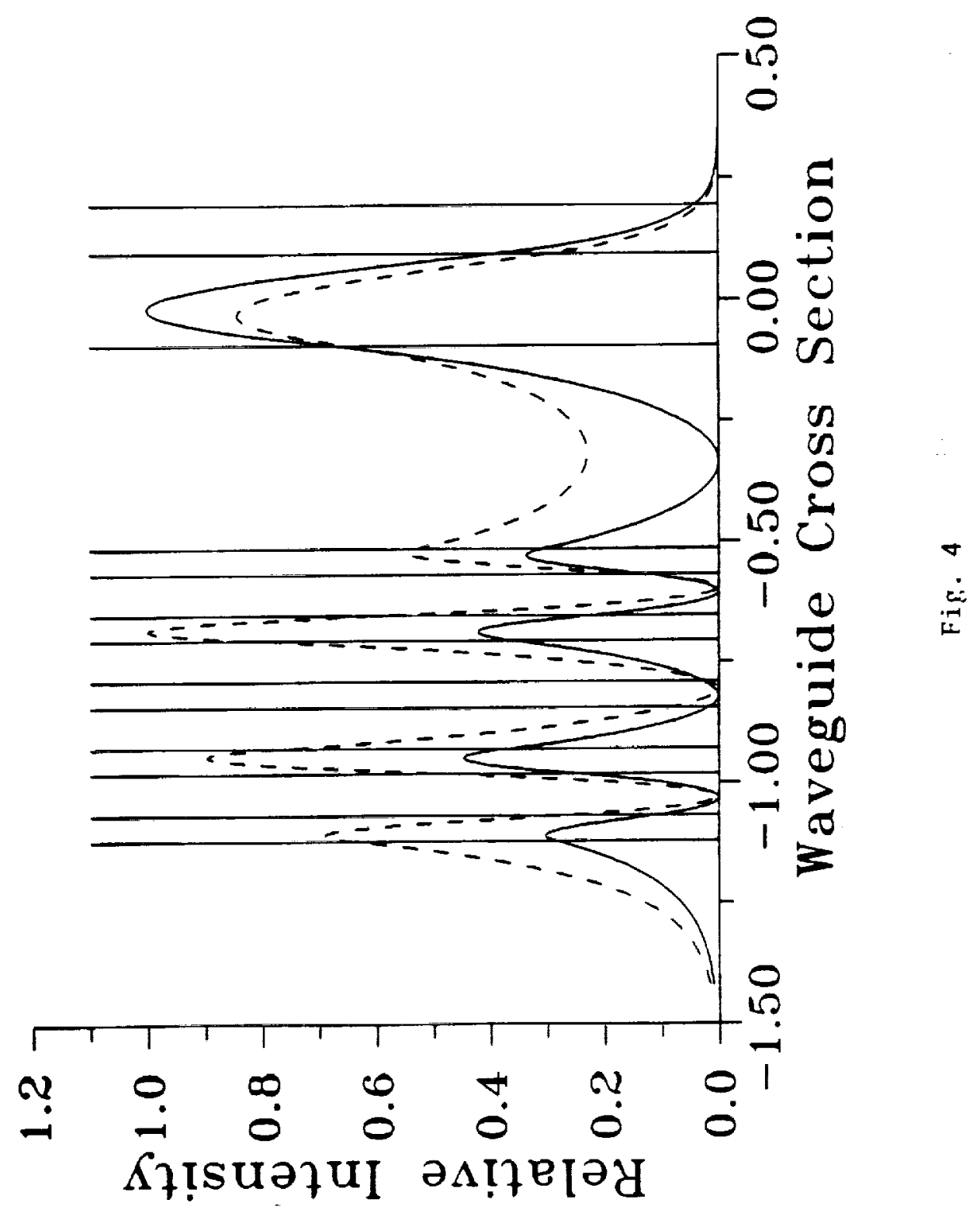




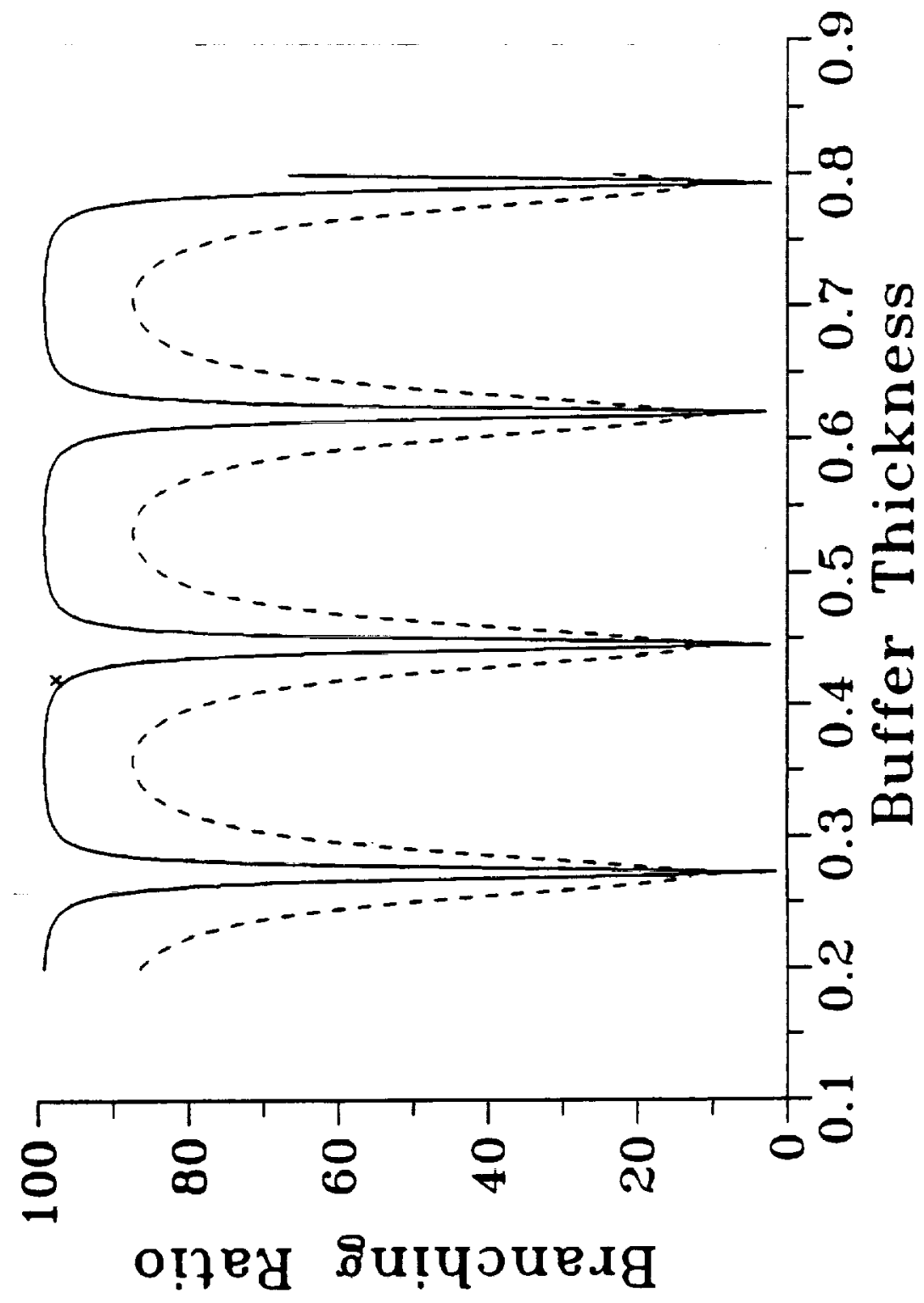

$\dot{\infty}$ 


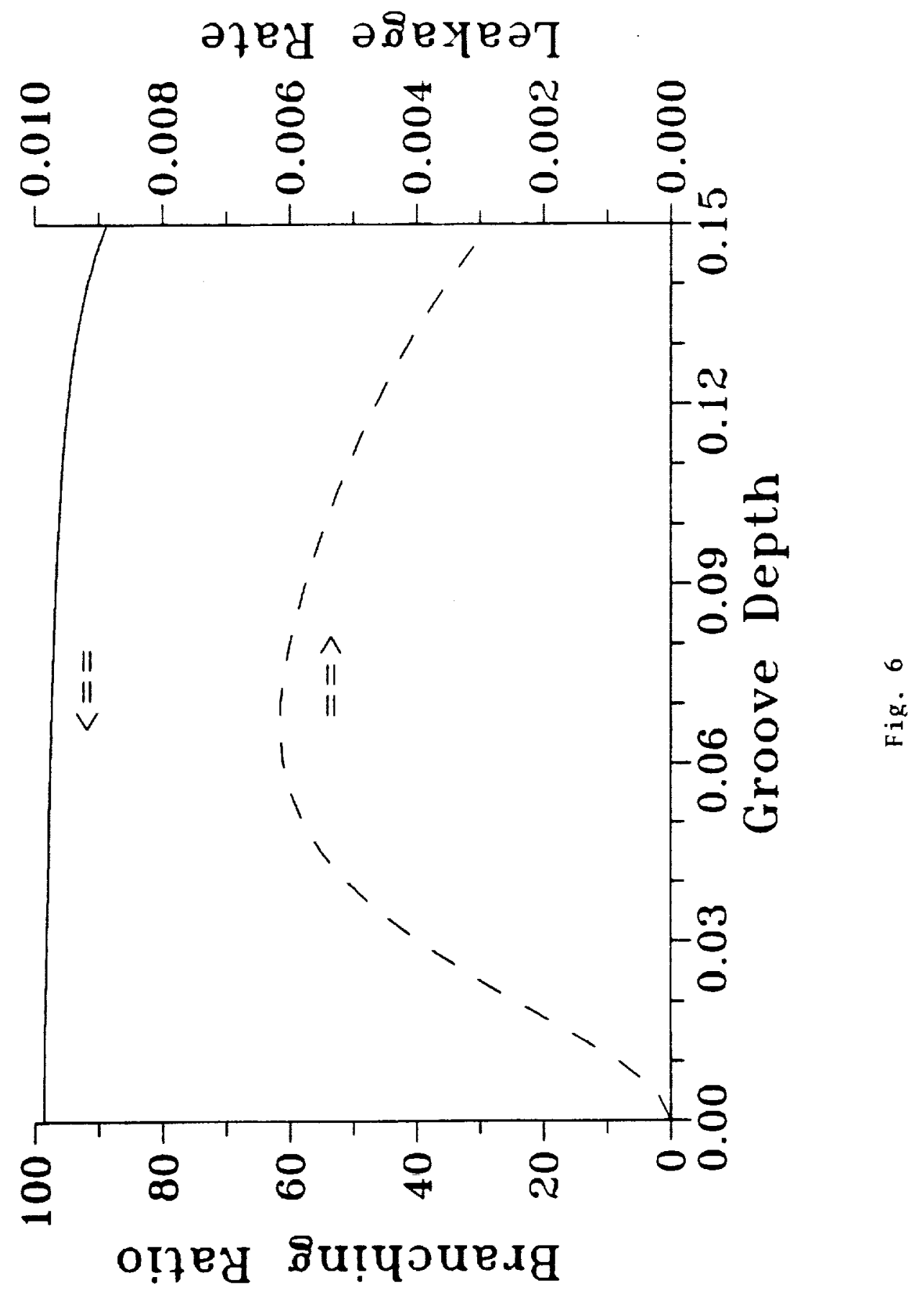




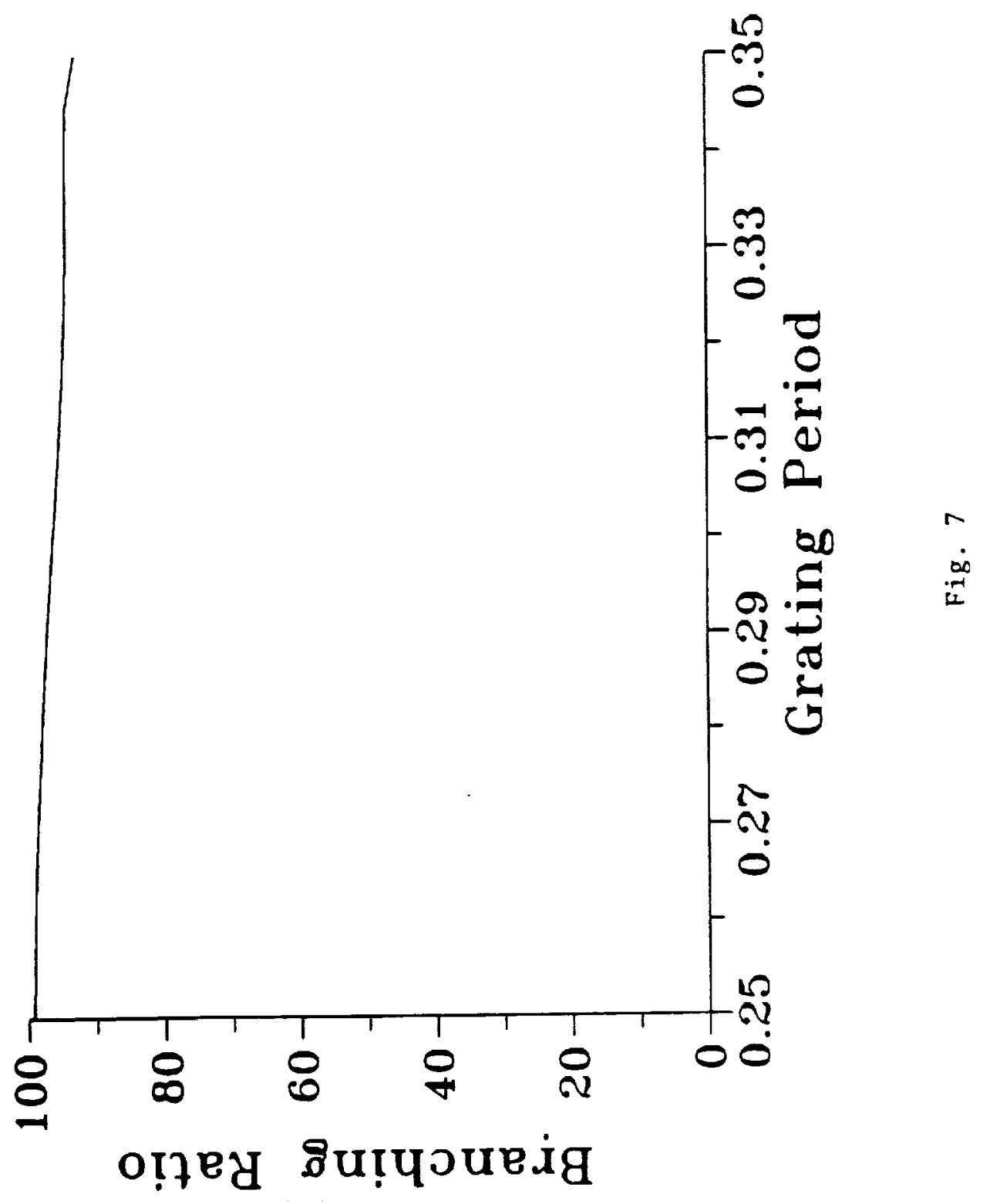




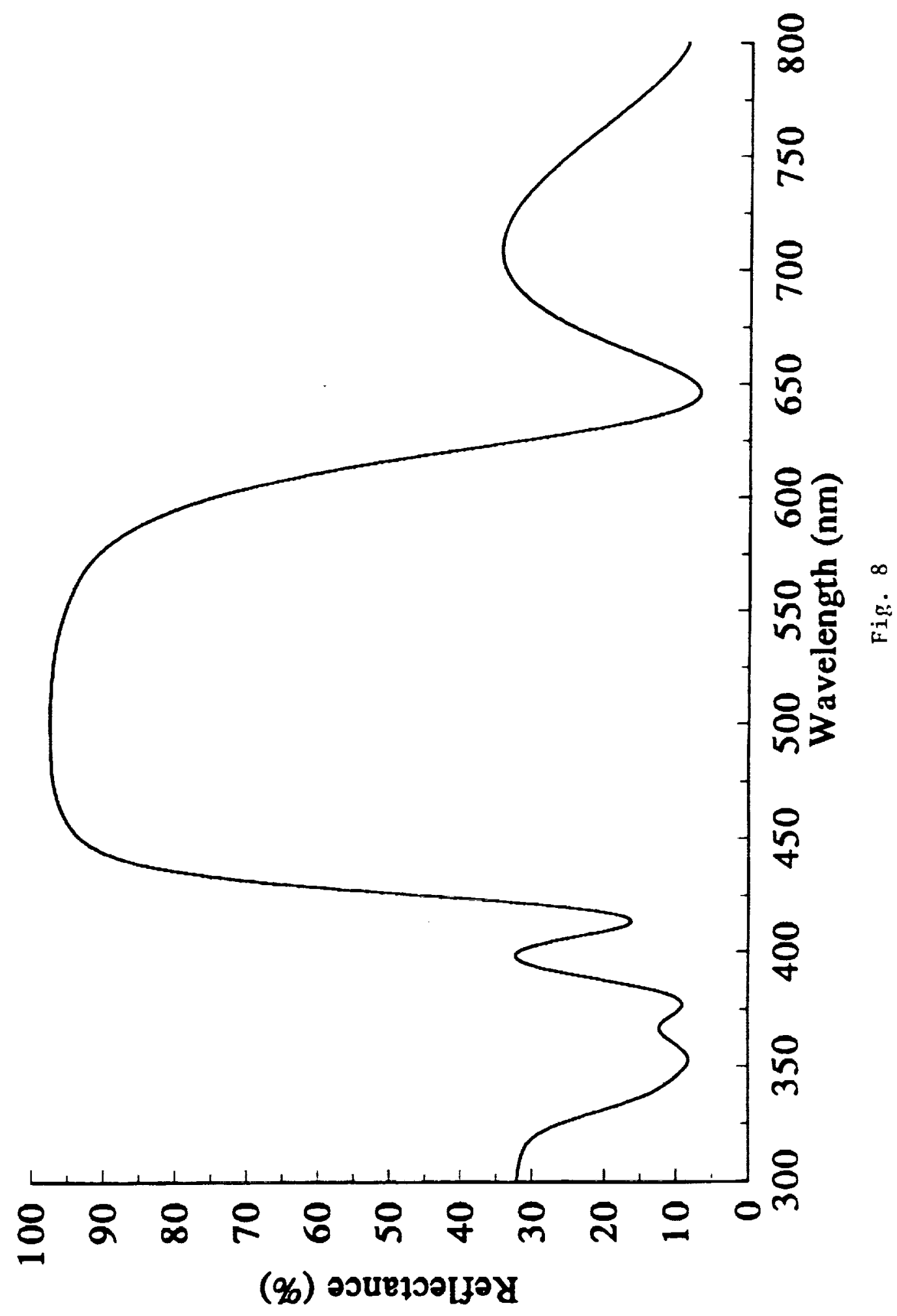




\section{APPENDIX N}

$\sim$

$\sim$ 
$=$

$\sigma$

$\sigma$

$\nabla$

$\varnothing$ 